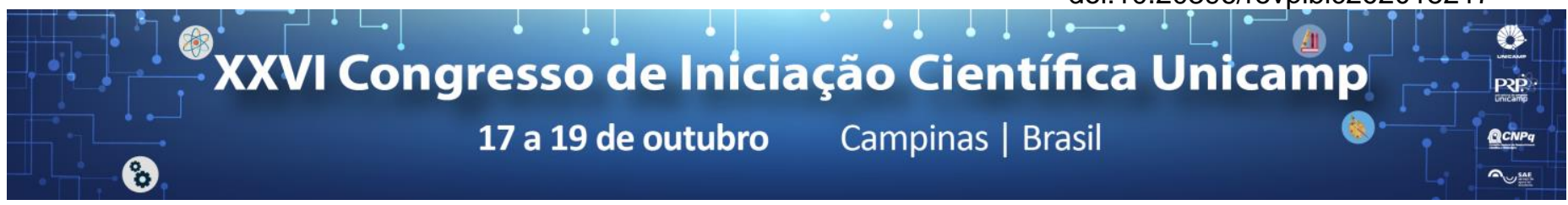

\title{
Study of NiCu multilayer magnetic nanowires
}

\section{Breno M. Cecchi ${ }^{\star}$ Kleber R. Pirota}

\begin{abstract}
Magnetic nanowires play an important role both in basic research, since they can exhibit new physical phenomena, and technological applications, such magnetic sotrage and magnetic sensors. It was investigated the magnetic properties of magnetic nanowires composed of multilayers made of $\mathrm{Ni}$ and $\mathrm{Cu}$, fabricated by electrodeposition method in alumina porous template. We obtained their magnetization curves at $2 \mathrm{~K}$ and $300 \mathrm{~K}$, and it was intended to perform magnetoresistance measurements. The latter was not done, because it was encountered difficulties in trapping the nanowires between electrodes.
\end{abstract}

\section{Key words:}

Nanomagnetism, nanowire, magnetoresistance.

\section{Introduction}

Magnetic nanowires are cylindric structures whose diameter is of the order of nanometers $\left(10^{-9} \mathrm{~m}\right)$, length of the order of micrometers $\left(10^{-6} \mathrm{~m}\right)$, and usually present at least one element among the ferromagnetic triplet $\mathrm{Fe}, \mathrm{Ni}$, Co, besides other possible transition metals. ${ }^{1}$ These samples are of interest both for basic research, because they can show physical phenomena that occur exclusively within the nanoscale, and for technological applications, such as magnetic sensors and high density magnetic recording media. ${ }^{1}$

The study objects were seven samples of magnetic nanowires made of several alternating layers of $\mathrm{Ni}$ and $\mathrm{Cu}$. They were fabricated by the traditional method of electrodeposition in alumina porous membranes. ${ }^{2}$ The objectives are to perform the magnetic characterization of the samples, through their hysteresis loop, to identify the process of magnetization reversal and to perform measurements of electrical transport, specifically magnetoresistance measurements.

\section{Results and Discussion}

The magnetic characterization was made by SQUID and VSM magnetometers at $2 \mathrm{~K}$ and $300 \mathrm{~K}$, so it was obtained the hysteresis loops of each sample. The interest parameters are shown in Tab. 1 , namely the coercivity $H_{c}$ and the saturation magnetic moment $m_{s}$. It is expected that both $H_{c}$ and $m_{s}$ decrease with temperature. This was indeed observed for all samples, except for PNWM_B. A more detailed study of the sample should be done for finding the reasons for this behaviour, as visualization of the nanowire structure and composition by some microscopy technique and micromagnetic simulations.

Table 1. Coercivity $H_{c}$ and saturation magnetic moment $m_{s}$ of each sample at $2 \mathrm{~K}$ and $300 \mathrm{~K}$.

\begin{tabular}{|c|c|c|c|c|}
\hline \multirow{2}{*}{ Sample } & \multicolumn{2}{|c|}{ Coercivity $\boldsymbol{H}_{\boldsymbol{c}}$ (Oe) } & \multicolumn{2}{c|}{ Saturation magnetic moment $\mathbf{~ m s}\left(\mathbf{1 0}^{\mathbf{- 4}} \mathbf{e m u}\right)$} \\
\cline { 2 - 5 } & $\mathbf{2 ~ K}$ & $\mathbf{3 0 0} \mathbf{~}$ & $\mathbf{2 ~ K}$ & $\mathbf{3 0 0 ~} \mathbf{~}$ \\
\hline PNWM_C & 412,2 & 206,4 & 9,3 & 6,8 \\
\hline PNWM_30.1 & 469,85 & 409,65 & 114 & 101 \\
\hline PNWM_1.2 & 298,25 & 248,5 & 44 & 39 \\
\hline PNWM_14.2 & 388,15 & 245,15 & 36,8 & 33,9 \\
\hline PNWM_B & 647,75 & 749,5 & 265 & 228 \\
\hline PNWM_16.2 & 394,2 & 288 & 28 & 7,5 \\
\hline PNWM_14.1 & 14,4 & 9,65 & 9,5 & \\
\hline
\end{tabular}

The magnetization curves give information about the whole nanowire matrix, while electrical transport measurements give information about an individual nanowire. To perform the latter, however, it is necessary to trap a unique nanowire between two electrodes. This can be accomplished by dielectrophoresis (DEP) method. ${ }^{3}$ It was encountered great difficulties in establishing the contact. We believe that the contact resistance between the nanowires and the electrodes is the principal responsible for this, ${ }^{3}$ and also may exist an insulating oxide coating in the $\mathrm{Cu}$ layers. By repeatedly performing DEP and changing parameters, we achieved a good contact for the sample PNWM_B. Unfortunately, the time employed in DEP was bigger than expected for this reason, and the magnetoresistance measurements were not made yet.

\section{Conclusions}

The magnetization curves are hysteresis loops as expected. The coercivities $H_{c}$ and the saturation magnetic moments $m_{s}$ decrease with temperature for all samples except for PNWM B, and we could not find an immediate explanation for this deviation, so a directed study should be done in this sense. We unfortunately did not perform magnetoresistance measurements because of the difficulty encountered in establishing contact between the nanowires and the electrodes, which we believe that was principally due to the contact resistance between them. Micromagnetic simulations together with magnetoresistance measurements can give information about the magnetization reversal mechanism dominant in the samples.

\section{Acknowledgement}

I would like to thank all LMBT/UNICAMP staff and students for the help and learning, and PIBIC/CNPq for the funding.

\footnotetext{
${ }^{1}$ GUIMARÃES, A. P. Principles of Nanomagnetism . Springer, 2009. 230 p. ${ }^{2}$ PIROTA, K. R. et al. Novel magnetic materials prepared by electrodeposition techniques: arrays of nanowires and multi-layered microwires. Journal of Alloys and Compounds, v. 369, p. 18-26, 2004.

${ }^{3}$ DOS SANTOS, M. V. P. et al. Dielectrophoretic manipulation of individual nickel nanowires for electrical transport measurements. Journal of Vacuum Science \& Technology B, v. 33, n. 3, 2015.
} 